\title{
Exploring pricing rules in combinatorial sealed-bid auctions
}

\author{
A. Mochon ${ }^{\text {a }, *}$, Y. Saez ${ }^{\text {b }}$ J.L. Gomez-Barroso ${ }^{a}$, P. Isasi ${ }^{b}$ \\ a Department of Applied Economics and Economic History, UNED, P Senda del Rey, 11, 28040 Madrid, Spain \\ b Department of Computer Science, Artificial Intelligence, Carlos III University, Av. Universidad 30, 28911 Leganes, Madrid, Spain
}

JEL classification:

C630

C730

D440

Keywords:

Core-selecting package auctions

Pricing rule

Co-evolutionary system

Agent-based model

Radio spectrum

\begin{abstract}
A B S T R A C T
The allocation of public goods such as the radio spectrum is a difficult task that the government must face. Currently, auctions are becoming an important tool to deal with this duty. In this context, the rules that the auctioneer establishes are particularly relevant, as the final outcome depends on them. When auctioning many related items, such as spectrum licenses, the bidders' values for one item may depend on the number of items already obtained (complements and substitutes items). In such circumstances, combinatorial auctions are the most appropriate alternative for allocating lots. This paper analyzes the implications of selecting a particular pricing mechanism on the final result in a combinatorial sealed-bid auction. The following pricing rules are selected: the first-price mechanism, the Vickrey-Clarke-Groves (VCG) mechanism, and the bidder-Pareto-optimal (BPO) core mechanism, a core-selecting auction. To test these pricing rules, a simulator of the auction model has been developed. Then, to tackle the complex problem of simulating bidders' behavior, a co-evolutionary system has been designed to identify improved strategies. The results revealed that the first-price mechanism yields inefficient outcomes and a notable reduction in the seller's revenues. Both the VCG and BPO mechanisms yield outcomes that are closer to the efficient allocation, and differences in revenues are affected by the presence of asymmetries.
\end{abstract}

\section{Introduction}

The radio airwave spectrum is a scarce resource for which licenses are allocated for its use by the government via competition or, increasingly, by auction. Depending on the service for which the spectrum is intended, each participant has the goal of obtaining a given amount of spectrum. Therefore, for participants, the licenses to be auctioned can be either substitutes (when a participant values a set of items less than the sum of the values of the individual items) or complements (when a participant values a set of items more than the sum of the values of the individual items); see Ausubel et al. (1997). In the presence of complementarities (synergies), bidders are interested in acquiring more lots to obtain their superadditive values. Nonetheless, it may be the case that the obtained spectrum is not sufficient to provide the expected service; therefore, if bidders win only some of the lots they require, then they can earn negative profits (exposure problem). To avoid such problems, the best option is to employ combinatorial auctions in which participants can bid freely for the combinations of lots in which they are interested. In this way, participants are encouraged to express their preferences fully.

Thus, despite their complexity, combinatorial auctions are becoming an essential tool when it comes to awarding multiple heterogeneous lots, particularly in the allocation of radio spectrum licenses (see Banks et al., 2003; Plott and Salmon, 2004, among others). When designing combinatorial auctions, as in any auction, it is essential to achieve an efficient allocation

\footnotetext{
* Corresponding author. Tel.: +34 913988876; fax: +34 913987821.

E-mail address: amochon@cee.uned.es (A. Mochon).
} 
and fair payments. ${ }^{1}$ Among other decisions, the price mechanism to be established is highly relevant. This paper focuses on the importance of choosing of a particular pricing rule for the final outcome in a combinatorial sealed-bid auction for heterogeneous goods. The good chosen is the radio-wave spectrum.

The following pricing rules have been selected for this comparison: the first-price (or pay-as-bid) mechanism, the Vickrey-Clarke-Groves (VCG) mechanism, and the bidder-Pareto-optimal (BPO) core mechanism, a core-selecting auction. Of these three pricing rules, the easiest to deploy and the most intuitive for participants is the first-price mechanism, simply because the winners have to pay what they bid. However, the main drawback of this mechanism is that participants have incentives to shade their bids. That is, when there is incomplete information about rivals' preferences, participants try to bid as little as possible, thus affecting the efficient allocation of the lots. An alternative is the VCG mechanism (see Vickrey, 1961; Clarke, 1971; Groves, 1973), whereby each winning bidder pays the opportunity cost for the units won. This mechanism is a generalization of the Vickrey second-price rule for a single item for combinatorial auctions, and its main advantage is that it is incentive compatible, i.e., that truth telling is the dominant strategy for all participants. However, this pricing rule also has important drawbacks (Ausubel and Milgrom, 2006). The main problem with the VCG mechanism is that the price finally paid by the winning bidders may not be a core allocation. In other words, there may be a coalition of bidders who would strictly prefer an alternative deal, including the seller. ${ }^{2}$ Therefore, in some cases, the initial result of the VCG mechanism is not socially acceptable.

In an effort to alleviate the problem generated by these two pricing rules, new methods have been developed that seek an efficient allocation capable of generating a core outcome. Within this subject, it is worth highlighting the work of Ausubel and Milgrom (2002), who proposed the ascending proxy auction. Later, Ausubel et al. (2006) developed the clock-proxy auction, and Avenali and Bassanini (2007) explored the first-price multiround combinatorial format. Also noteworthy is the ascending-price combinatorial auction called iBundle, which has been studied, among others, by Parkers and Ungar (2002) and by Mishra and Parkes (2007). In this same line of work, Hoffman et al. (2006) conducted a comparison of different proxy methods to obtain core outcomes. Avenali (2009) developed the threshold-price rule as an alternative capable of determining the highest losing bids in combinatorial auctions.

Finally, Day and Milgrom (2008) proposed the core-selecting package auction as a mechanism that selects core allocations with respect to reported values. In this mechanism, the auctioneer determines the combination of feasible bids that maximizes his revenue and establishes a pricing rule that assures a core outcome respecting to the submitted bids. Several pricing rules have been developed for core-selecting auctions. Day and Raghavan (2007) proposed an approximate VCG mechanism that generates BPO core outcomes in any combinatorial sealed-bid auction. These authors suggest a mechanism by which the winning bidders receive a discount on their bids, but the auction result itself is always a core allocation with respect to submitted bids. With this pricing rule, the bidder-optimal core allocation minimizes the maximum deviation from the VCG payments, and thus, the auction minimizes the maximal gain from deviating from truthful bidding (Day and Milgrom, 2008). Furthermore, Day and Cramton (2008) presented the quadratic-core-pricing rule and Erdil and Klemperer (2010) defined a class of payment rules called reference rules to make core-selecting package auctions more robust. The pricing rule that we selected in this paper to test the core-selection auction outcome is that presented by Day and Raghavan (2007), i.e., the BPO mechanism.

Theoretical analysis of package auctions in the presence of complementarities with incomplete and asymmetric information can be extremely difficult to explore. Hence, most research has been limited to complete-information models. Nevertheless, some authors have started to compare core-selection auction outcomes considering incomplete-information games but with simple models. Goerre and Lien (2009) developed a model with two goods, a global bidder and two local bidders whose values are privately known, independent and uniformly distributed. These authors solved for the Bayesian-Nash equilibrium of the core-selecting auction with the nearest Vickrey pricing rule and found that the VCG mechanism dominates it in expected revenues and efficiency. They claimed that the rationale for this result is that bidders tend to shade their bids when the nearest Vickrey pricing rule is implemented.

Ausubel and Baranov (2010) compared some core-selecting auctions ${ }^{3}$ as well as the VCG mechanism and the first-price rule in a model with two goods, two local bidders and one global bidder. They considered an incomplete-information model and performed a full equilibrium analysis assuming independent values as well as positive correlated values. These authors found that, for the independent case, the VCG mechanism obtained higher revenues and realized greater efficiency than the best core-selecting auction analyzed, because the core-selecting payments incentivize bidders without an intersection of interest to underbid. Nevertheless, when values are positively correlated, a core-selecting auction yields higher revenues and realized the same efficiency as does the VCG mechanism. These authors found that the choice between a core-selecting auction and the VCG mechanism is sensitive to the information structure.

The current paper seeks to advance the analysis of package auctions for more complex models simulating real environments for which theoretical results are not available. As Roth (2002) pointed out, economists play a key role in market design,

\footnotetext{
1 Porter et al. (2009) tested the combinatorial sealed-bid auction, the sequential English clock auction, and the combinatorial English clock auction in terms of revenue maximization and efficiency.

2 The next section explains this concept through an example.

3 The core-selecting auctions analyzed are the following: nearest-Vickrey payments, proxy auction payments, proportional pricing payments and nearestbid payment.
} 
and both experimental and computational economics are perfect complements to game theory in environments that elude analytical solutions. Hence, our aim is to use computational methods to fill the gap between theory and design in an effort to understand how to deal with the complexities of real markets.

We considered a model with incomplete information and different degrees of asymmetries. In each scenario, we have three or four bidders and six items. Bidders have intersection of interest, a complex preference structures (lots can be both substitutes and complements) and can submit as many bids as they want for each lot or combination. ${ }^{4}$ To perform the analysis, first, a simulator of the auction model was developed in which the three pricing rules have been implemented. To simulate the behavior of the participants, we executed a co-evolutionary system based on populations in which a Genetic Algorithm (GA) seeks to maximize the profit of each agent, i.e., the difference between each participant's valuation and the price he/she pays. The strategies for each agent against the others co-evolved over the 1000 iterations, generating improved strategies for each bidder.

Our results reveal that, in a combinatorial sealed-bid auction, with the first-price rule bidders tend to shade their reported values, and thus, this mechanism yields the lowest revenues and inefficient outcomes. Results comparing the VCG mechanism and the core-selecting auction implementation are not as clear. When bidders are symmetric, the core-selecting auction with the BPO mechanism yields higher revenues than does the VCG, but the opposite happens when bidders are asymmetric. Nevertheless, when we focus our attention on those auctions in which the BPO identifies a significant number of blocking coalitions, ${ }^{5}$ the core-selection auction always yields higher revenues than does the VCG mechanism, even in the presence of asymmetries.

This paper is structured as follows: Section 2 details the selected auction model and the three pricing rules we implemented. Section 3 discusses the characteristics of the simulated radio-wave spectrum auction. Three different scenarios were modeled, one with symmetric participants and the other two with different degrees of asymmetry among the participants. Section 4 explains the methodology used to simulate the behavior of the participants through a co-evolutionary system. The results for each pricing rule are presented and compared in Section 5. Finally, Section 6 is devoted to discussing the conclusions of this study and potential future lines of work.

\section{The auction and pricing rules}

Let us consider an auction with $N$ lots available to award among $M$ participants. Each lot of the set $I=\{1,2, \ldots, N\}$ is represented by the letter $i$ and each of the participants $J=\{1,2, \ldots, M\}$ by the letter $j$. For each combination of lots $S \subseteq I, v_{j}(S)$ represents the valuation of participant $j$ for the combination $S$ (the maximum amount he/she would be willing to pay). In a combinatorial sealed-bid auction, each participant submits as many bids as he/she likes for the lots, or combinations of lots, in which he/she is interested in a single round. The bid submitted (reported value) by participant $j$ for combination $S$ is represented by $b_{j}(S)$. We assume an XOR bidding language, i.e., winning bidders can only win a single bid, bids are mutually exclusive.

Given all the bids submitted by the participants, the seller determines the combination of feasible bids (in which no item is awarded more than once) that maximizes his revenue, i.e., that solves the Winner Determination Problem (WDP) ${ }^{6}$ according to Eq. (1):

$$
\begin{aligned}
\max \sum_{j \in J} \sum_{S \subseteq I} b_{j}(S) x_{j}(S) & \\
\text { subject to } & \sum_{S \supseteq\{i\}} \sum_{j \in J} x_{j}(S) \leq 1 \quad \forall i \in I, \\
& \sum_{S \subseteq I} x_{j}(S) \leq 1 \quad \forall j \in J, \\
& x_{j}(S) \in\{0,1\} \quad \forall S \subseteq I, \quad \forall j \in J .
\end{aligned}
$$

where $x_{j}(S)$ is a binary variable that is equal to one if and only if bidder $j$ is awarded bundle $S \subseteq I$. Solving the WDP is an NP-complete problem (Sandholm, 2002). Hence, advanced computational techniques must be used to solve or approximate this task. In this work, we have decided to use an $\mathrm{A}^{*}$ based on a branch on bids (BOB) formulation search algorithm. The BOB formulation algorithm uses a tree representation with the possible combination of bids. Each node of the tree is a possible

\footnotetext{
${ }^{4}$ There are 23 possible bids per bidder.

${ }^{5}$ The comparison includes experiments in which both the sincere bidding case of the BPO and the co-evolutionary system of the BPO identifies simultaneously three or more blocking coalitions.

${ }^{6}$ We use the formulation presented by Day and Raghavan (2007).
} 
Table 1

Table of bids (Euros).

\begin{tabular}{lllll}
\hline & $b_{1}(S)$ & $b_{2}(S)$ & $b_{3}(S)$ & \\
\hline A & $20^{\mathrm{a}}$ & 10 & $b_{4}(S)$ & 10 \\
$\mathrm{~B}$ & & $20^{\mathrm{a}}$ & 10 & $20^{\mathrm{a}}$ \\
$\mathrm{C}$ & 10 & & 10 & 28 \\
$\mathrm{AB}$ & & & & 28 \\
\hline
\end{tabular}

a The winners after solving the WDP.

combination of bids, so the $\mathrm{A}^{*}$ algorithm ${ }^{7}$ searches for the combination that solves the WDP (for more details about this implementation, see Saez et al., 2008).

After establishing the winning combination to solve the WDP, participants have to pay for the lots obtained. The final payment depends on the pricing rule established by the seller. The variable $p_{j}$ indicates the amount payable by the winning participant $j$, and the superscripts are used to differentiate between the different pricing rules used in this work: $p_{j}^{1 \text { st }}, p_{j}^{V C G}$ and $p_{j}^{B P O}$. The seller's final revenue is equal to the sum of the payment of all winning bidders, as per Eq. (2):

$$
R=\sum_{j \in W} p_{j},
$$

where $W$ is the set of winning participants. Superscripts are also included to differentiate between revenues for each pricing rule: $R^{1 \mathrm{st}}, R^{V C G}$ and $R^{B P O}$.

\subsection{First-price mechanism}

If the seller establishes a first-price (or pay-as-bid) pricing rule, each participant will pay an amount equal to the bid made: $p_{j}^{1 s t}=b_{j}\left(S_{j}^{*}\right)$, where $S_{j}^{*}$ denotes the combination of lots that participant $j$ has won.

Example: Suppose there is a combinatorial sealed-bid auction with three lots and four participants who made their bids as indicated in Table 1.8

The winning combination of bids that maximizes the seller's revenues, i.e., the solution to the WDP, is equal to $b_{1}\left(A^{*}\right)+$ $b_{2}\left(B^{*}\right)+b_{3}\left(C^{*}\right)=20+20+20=60$ Euros. Therefore, the first participant wins lot A, the second lot B, and the third lot C. The price that each participant will pay is equal to $p_{1}^{1 \mathrm{st}}=p_{1}^{1 \mathrm{st}}=p_{3}^{1 \mathrm{st}}=20$ Euros. In this example, the revenues will be equal to $R^{1 \text { st }}=60$ Euros.

This pricing rule is simple to implement, and it reduces the risk of collusion among participants while fostering their participation. The main problem with this rule is that participants tend to shade their bids, a strategy that affects the final allocation of the lots.

\subsection{Vickrey-Clarke-Groves mechanism}

With the VCG mechanism, the price paid by the winning participant $j \in W$ is equal to the opportunity cost of the items won. This amount depends only on the bids made by the winning bidder's rivals and is calculated according to Eq. (3):

$$
p_{j}^{V C G}=\alpha_{j}-\sum_{k \neq j} b_{k}\left(S_{k}^{*}\right)
$$

where $\alpha_{j}=\max \left\{\sum_{k \neq j} b_{k}\left(S_{k}\right) \mid \sum_{k \neq j} S_{k} \leq I\right\}$ is the result of solving the WDP again using all the bids made by all participants except participant $j$. The second term of Eq. (3) is equal to the sum of the winning bids made by all participants except $j$.

Example: Continuing with the table of bids in the previous example (Table 1), the allocation of lots remains constant $\left(b_{1}\left(A^{*}\right), \quad b_{2}\left(B^{*}\right), \quad b_{3}\left(C^{*}\right)\right)$; however, the prices to be paid will be different.

To calculate the payment that the first winning participant would have to make, the first participant's bids are ignored, and the WDP is recalculated, yielding $\alpha_{1}=b_{4}(A)+b_{2}(B)+b_{3}(C)=10+20+20=50$ Euros; see Table 2. The second term of Eq. (3) for the first participant is equal to $\sum_{k \neq 1} b_{k}\left(S_{k}{ }^{*}\right)=b_{2}\left(B^{*}\right)+b_{3}\left(C^{*}\right)=20+20=40$. Therefore, the final payment that the first participant will have to make for lot $A$ will be equal to $p_{1}^{V C G}=50-40=10$ Euros. Final payments are calculated for the other winners in the same way: $p_{2}^{V C G}=p_{3}^{V C G}=10$ Euros. Under this pricing rule, the total revenue the seller obtains is equal to $R^{V C G}=30$ Euros.

\footnotetext{
7 The $\mathrm{A}^{*}$ algorithm (A-Star) is a best-first search algorithm that explores a graph by expanding the most promising node chosen according to a specified rule. It usually uses a distance-plus-cost heuristic function to determine the order in which the search visits nodes in the graph. It is widely used in pathfinding, in combinatorial search and in graph traversal. For more information, see Hart et al. (1968).

8 With three lots, there are a total of seven combinations, but for simplicity, in this example, only four combinations have been considered.
} 
Table of bids ignoring those made by the first participant (Euros).

\begin{tabular}{llll} 
& $b_{1}(S)$ & $b_{2}(S)$ & $b_{3}(S)$ \\
A & 10 & $b_{4}(S)$ & 10 \\
B & $20^{\mathrm{a}}$ & 10 & $20^{\mathrm{a}}$ \\
$\mathrm{C}$ & & 10 & 28 \\
$\mathrm{AB}$ & & & 28 \\
\hline
\end{tabular}

a The winner after solving for WDP ignoring the bids of the first participant.

The main advantage of the VCG mechanism is that it is incentive compatible, i.e., that truthful bidding is a dominant strategy for each bidder. Therefore, if participants follow this strategy, then an efficient allocation of the lots is achieved.

\subsection{Core-selecting auction: the bidder-Pareto-optimal core mechanism}

Among core-selecting package auctions, the BPO mechanism developed by Day and Raghavan (2007) offers an efficient solution for making socially acceptable payments in any combinatorial sealed-bid auction. To accomplish this goal, the mechanism works off the same efficient allocation of lots that the VCG mechanism establishes, but it imposes restrictions on payments so that they are updated in successive iterations $\left(p_{j}^{t}\right)$ until a result in the core respect to reported values is achieved. The final price paid by each winning participant is calculated by the following steps:

1. The method begins with a vector of payments $p_{j}^{t}$, and the starting vector corresponds to the one obtained with the VCG mechanism: $p_{j}^{1}=p_{j}^{V C G}$.

2. To determine whether there is a group of participants willing to offer an amount greater than the sum of the current payments $\sum_{j \in W} p_{j}^{t}$, one must calculate how much the participant $j$ would be willing to provide to the coalition in each iteration $t$. The coalitional contribution $q_{j}$ is calculated as follows:

a. The participant $j \in W$ who has won lot $S_{j}^{*}$ will not want to make a contribution that will report a benefit smaller than the current one $b_{j}\left(S_{j}^{*}\right)-p_{j}^{t}$, which is his/her opportunity cost. Therefore, the coalitional contribution for each combination $S_{j}$ at iteration $t$ is equal to $q_{j}\left(S_{j}, p_{j}^{t}\right)=b_{j}\left(S_{j}\right)-\left(b_{j}\left(S_{j}^{*}\right)-p_{j}^{t}\right)$, where $S_{j}$ refers to any combination of lots that can match with $S_{j}^{*}$.

b. The participant $j \notin W$ who has not won a lot has no opportunity cost, and so the participant's contribution to the coalition coincides with the initial bid made for each combination of lots $q_{j}=b_{j}(S)$.

3. Given the coalitional contributions from all participants, the WDP is calculated. In other words, the highest coalitional value relative to the current payments, and the coalitional value $z\left(p^{t}\right)$ is obtained in the iteration $t$.

4. If there is no coalition that can report higher profits to the seller than the sum of the current payments, i.e., if $\sum_{j \in W} p_{j}^{t}=$ $z\left(p^{t}\right)$, then this coalition does not block the efficient allocation and the auction closes at the current proposed unblocked core outcome $p^{t}$.

On the other hand, if this coalition blocks the current allocation - that is, if $\sum_{j \in W} p_{j}^{t}<z\left(p^{t}\right)-$ there is a group of participants $C^{t}$ that offers a solution in which the members of the coalition and the seller improve. To avoid this situation (which would be socially unacceptable), the payments of the winning participants are recalculated by applying the core constraint defined in Eq. (4).

$$
\sum_{j \in W \backslash C^{t}} p_{j} \geq z\left(p^{t}\right)-\sum_{j \in W \cap C^{t}} p_{j}^{t}
$$

where $\sum_{j \in W \backslash C^{t}} p_{j}$ denotes the payments of the winning participants that do not belong to the coalition, and $\sum_{j \in W \cap C^{t}} p_{j}^{t}$ denotes the payments of the winning participants that do form part of the coalition.

5. Finally, the new BPO core payments $p^{t+1}$ are calculated and the process is re-initiated. ${ }^{9}$ This iterative process continues to increase the payments made by the winning participants until an unblocked core outcome is reached.

For those cases in which there is no blocking coalition, the vector of payments established by the VCG mechanism will coincide with that of BPO : $p_{j}^{V C G}=p_{j}^{B P O}$.

Example: If we use the same data as in the previous example (Tables 1 and 2), the vector of payments of iteration $t=1$ coincides with the VCG vector of payments $p_{j}^{1}=p_{j}^{V C G}$, with their sum equaling $\sum_{j \in W} p_{j}^{1}=30$ Euros. To determine whether there is a coalition that can block this result, the coalitional contribution of each participant $q_{j}\left(S_{j}, p_{j}^{1}\right)$ is calculated for this iteration (see Table 3). We calculate the WDP with these values, and the coalitional value is obtained for the first iteration

\footnotetext{
${ }^{9}$ Multiple optimal BPO prices may exist; we follow the linear program suggested by Day and Raghavan (2007), which produces equitable BPO core payments.
} 
Table 3

Coalitional contribution for $t=1$ (Euros).

\begin{tabular}{llccc}
\hline & $q_{1}\left(S_{1}, p_{1}^{1}\right)$ & $q_{1}\left(S_{2}, p_{2}^{1}\right)$ & $q_{3}\left(S_{3}, p_{3}^{1}\right)$ & $q_{4}\left(S_{4}, p_{4}^{1}\right)$ \\
\hline A & 10 & 0 & & 10 \\
B & 10 & 10 & 0 & 10 \\
C & 0 & & $10^{\text {a }}$ & 10 \\
AB & & & & $28^{\mathrm{a}}$ \\
\hline
\end{tabular}

a The highest coalitional value relative to the current payments.

Table 4

Coalitional contribution for $t=2$ (Euros).

\begin{tabular}{lccrr}
\hline & $q_{1}\left(S_{1}, p_{1}^{2}\right)$ & $q_{1}\left(S_{2}, p_{2}^{2}\right)$ & $q_{3}\left(S_{3}, p_{3}^{2}\right)$ & $q_{4}\left(S_{4}, p_{4}^{2}\right)$ \\
\hline A & 14 & 0 & & 10 \\
B & 14 & 14 & 0 & 10 \\
C & 0 & & 10 & 10 \\
AB & & & & 28 \\
\hline
\end{tabular}

$z\left(p^{1}\right)=q_{4}(A B)+q_{3}(C)=28+10=38$ Euros. Because $\sum_{j \in W} p_{j}^{1}=30<z_{C^{1}}\left(p^{1}\right)=38$, the VCG outcome is not in the core, and $C^{1}$ acts as a blocking coalition. Therefore, to calculate the new BPO payments, the restriction $p_{1}^{2}+p_{2}^{2} \geq 38-10$ is included. The new payments at the iteration $t=2$ are equal to $p_{1}^{2}=14$ Euros, $p_{2}^{2}=14$ Euros, and $p_{3}^{2}=10$ Euros. With this vector of payments, the coalitional contribution is recalculated for each participant at the iteration $t=2 q_{j}\left(S_{j}, p_{j}^{2}\right)$, the values of which are shown in Table 4 . When calculating the WDP with these new contributions, we obtain $\sum_{j \in W} p_{j}^{2}=z\left(p^{2}\right)=38$ Euros. In other words, in this iteration, no other coalitions exist that block the current payments; thus, the core outcome has been reached and the process ends. In implementing the BPO mechanism, the seller obtains revenues equal to $R^{B P O}=38$ Euros.

The main objective of the BPO mechanism is to provide an efficient allocation of the lots along with fair payments, i.e., a core outcome with respect to reported values for any combinatorial auction.

\section{Scenarios}

The three pricing rules were compared in the context of a real auction: the auction for the allocation of licenses for radio-wave spectrum use. Specifically, we simulated the sale of a portion of so-called digital dividend, which is the "surplus" spectrum in the transmission of television signals after the switchover from analog to digital broadcasting by terrestrial waves, as the latter technology is much more efficient in terms of spectrum. These auctions have already been conducted in countries such as the U.S. and Germany. The simulation we carried out was endowed with characteristics that could be representative of any European country of medium to large size where bidders have intersection of interest. This choice lends realism to the model, yielding more relevant results.

In this exercise, we consider the digital dividend (the total spectrum to be auctioned) to correspond to $80 \mathrm{MHz}$, which is divided into three segments of 24, 32, and $24 \mathrm{MHz}$. Each segment is apportioned into the following lots: $1 \mathrm{lot}$ of $24 \mathrm{MHz}$ (lot A), 2 lots of $16 \mathrm{MHz}$ (lots B, B), and three lots of $8 \mathrm{MHz}$ (lots C, C, C), resulting in 23 possible combinations (packages).

With no pre-allocation of the spectrum to a particular service, companies with very diverse backgrounds and levels of market power would participate. Regarding the source, both broadcasters and mobile operators would bid for the spectrum. With regard to market power, in every market the potential participants range from established operators that account for a significant share in their markets to new companies hoping to enter these markets. This diversity sets up a general scenario with strong asymmetries. Nevertheless, for the purposes of this study, it is interesting to consider not only a scenario with strong asymmetries but also other scenarios with the participation of symmetrical bidders or with less pronounced asymmetries. For this reason, we created three individual scenarios in which the number and type of potential participants are restricted as follows.

\subsection{1st scenario: symmetric bidders}

In this scenario, there are three participants ${ }^{10}$ in the auction whose values are distributed according to the same distribution function, i.e., symmetric bidders. They all represent leading TV broadcasters belonging to a major media company, and they attend the auction with similar conditions and interests: a greater spectrum in which to broadcast new channels in standard or high definition. Considering their assessments of the spectrum (see below), they would be qualified as "average bidders" in an unrestricted auction.

\footnotetext{
10 These participants correspond to bidders B1, B2, and B3 in Table 6.
} 


\subsection{2nd scenario: asymmetric bidders}

In the second scenario, there are four participants ${ }^{11}$ : two average participants (two leading TV broadcasters as in the scenario above) and two strong ones. These two strong bidders are incumbent mobile operators interested in strengthening their current service offerings, particularly in terms of the transmission of data at high speed (wireless broadband), and even new services.

\subsection{3rd scenario: strong asymmetries}

In this scenario, there are also four participants ${ }^{12}$ : two strong (the two incumbent mobile operators in the previous scenario) and two weak (alternative mobile operators, with smaller market shares, lower deployed infrastructure and fewer resources.)

In all scenarios, participants have private information about their values. The spectrum valuation made by each type of participant was determined from the estimates provided by the British regulator (Ofcom) to identify the incremental producer and consumer value generated from the use of spectrum (Ofcom, 2006). To obtain the profile of each bidder, these values were modified taking into account the characteristics of the markets themselves, the bidder's position in the market (incumbent/leading, established alternative operator/secondary or new entrant) and by assigning each participant an expectation of future profitability of those markets (optimistic, conservative, or unenthusiastic). Given these conditions, for each agent a range of values is obtained for a number of spectrum lots that are considered "optimal" (amounts that vary depending on the services intended to be dedicated to the spectrum). In turn, these values are tailored to each lot and combination of lots, considering the possible effects of substitution or complementarity. ${ }^{13}$

\section{Experimental procedure}

Modeling the behavior of participants in auctions is not a simple task. One approach to study this type of games is to use biologically inspired techniques. The vast majority of studies have proposed applications of evolutionary computation techniques for identifying appropriate trading strategies in auctions. An early work in this line was an evolutionary simulation among trading agents submitted to the Santa Fe Institute Double Auction Tournament, Rust et al. (1993). Andreoni and Miller (1995) developed a GA to capture the bidding patterns evident in first- and second-price auctions. Cliff (1998) applied GAs to evolve improved versions of his Zero Intelligent Plus agents for continuous double auction trading strategies. Dawid (1999) developed a two-population GA to study the learning behavior of buyers and sellers in a sealed-bid double auction. Wen and David (2001) addressed the problem of constructing optimal bidding strategies for competitive suppliers in a day-ahead energy market using a GA. Focusing on auction mechanisms, Byde (2002) used GAs with a multiagent system to compare the standard first-price and second-price sealed-bid auctions. In that work, for each scenario (auction setting and distribution of private and common values), the GA was applied to evolve a trading strategy. Then, the fitness function evaluated the average revenue of the given auction with respect to a population of traders using that strategy. Similarly, Cliff (2003) extended his line of research on evolving trading strategies and auction rules to evaluate aggregate market performance, and Saez et al. (2007) used independent parallel GAs to study the outcome of Ausubel auctions with different rationing rules. Finally, the use of GAs for analyzing the clock-proxy auction is covered in Mochon et al. (2011).

In this type of game, where the benefit to one participant depends on the behavior of others, co-evolutionary techniques are preferable to evolutionary ones; see Hillis (1990). In fact, such techniques have been successfully applied for similar behavior in models of societies (Lund, 1995), learning problems of game theory (Rosin and Belew, 1995 or Miller, 1996), and market models (Chen et al., 2006), among others.

Co-evolutionary techniques arise in response to the need for an evolutionary algorithm that can learn from its own experience, without being trained by humans. The algorithm's performance has been demonstrated from the empirical and theoretical points of view, as shown in Tivnan (2005). When applied to auctions, this type of learning technique has some key advantages over others: the participants' behavior can be implemented in a natural and intuitive way in which each agent (participant) is considered a species, and each species has a population of independent strategies. This scheme allows each agent or species to display an effect similar to the memory among the various iterations of auctions, something very interesting in this type of iterated games, see Yao and Darwen (2000) or Mochon et al. (2007). The idea of introducing different species that co-evolve as ecosystems of nature is not new: see Hillis (1990), Husbands and Mill (1991), Potter and De Jong (1995) or Giordana and Neri (1996). This technique is an elegant idea inspired by the origins and biological evolution of species in an environment. Other approaches exist that utilize co-evolutionary techniques; however, none have been done with combinatorial auctions. In addition, all scientific papers on (non-combinatorial) auctions have focused on implementing genetic programming-based solutions instead of GAs. For instance, see Phelps et al. (2002), who applied a co-evolutionary genetic programming system to the development of double auctions in a wholesale electricity marketplace;

\footnotetext{
11 Strong participants correspond to bidders B1 and B2, whereas the average participants correspond to B3 and B4 in Table 6 .

12 Strong participants correspond to bidders B1 and B2, whereas the weak participants correspond to B3 and B4 in Table 6 .

13 See Gomez-Barroso et al. (2011).
} 


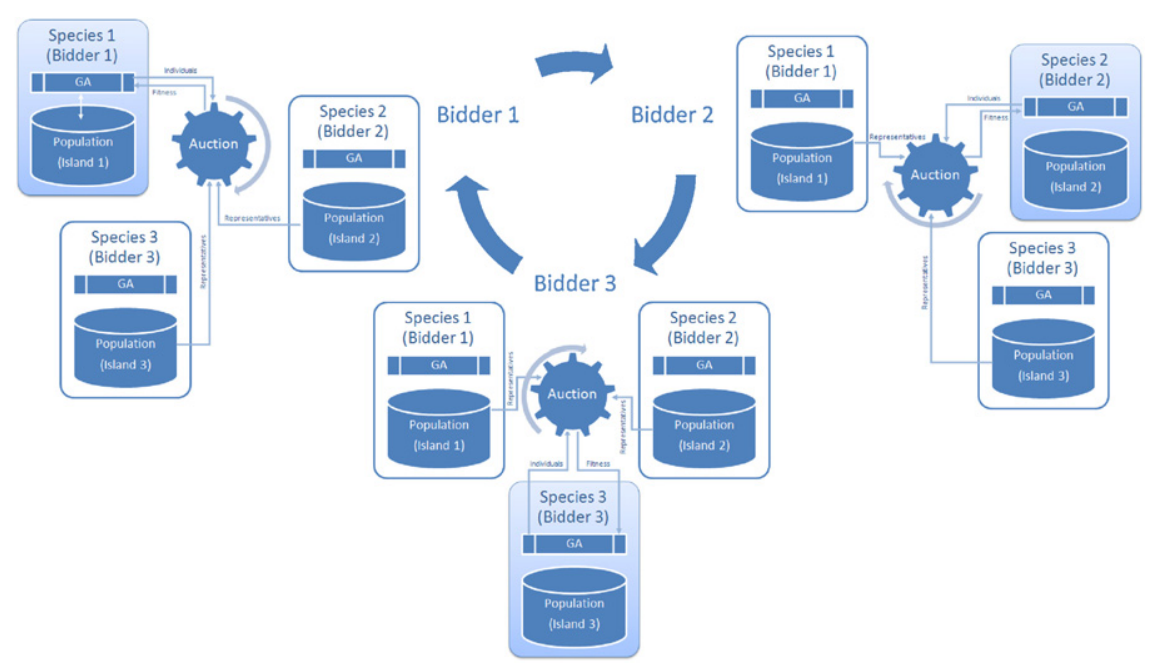

Fig. 1. General scheme of the co-evolutionary system performance (symmetric scenario: 3 agents).

Becker and Seshadri (2003), who described how cooperative co-evolution can be used to generate trading rules with genetic programming; more recently, Nie et al. (2008), who demonstrated a co-evolutionary computation method to validate reverse multi-attribute auction settings with one buyer and many sellers and, finally, Chen et al. (2009a, 2009b), who published two works on the dynamics of trader behaviors using a co-evolutionary genetic programming system to simulate and analyze a double auction market.

\subsection{The co-evolutionary system proposed}

The overall design of the co-evolutionary system proposed in this work is very similar to the one reported by Potter and De Jong (2000). Because all agents seek to maximize their profits (fitness value), each of them implements a GA that tries to achieve this objective. However, unlike the work of Potter and De Jong (2000), in this case, the best valued strategy is always chosen to represent each species (candidate or representative strategy); i.e., to assess a species, all of its population strategies are evaluated against the best strategies of the other participants. It would be interesting to evaluate these strategies against all strategies of all agents (as Potter and De Jong, 2000, have done), but the number of combinations increases exponentially, making the implementation of this approach unfeasible from a computational point of view (each evaluation requires to simulate an auction). Therefore, the evaluation of strategies for each species will be based on the best individuals (representatives) of the other species during the auctions.

Because each species implements an independent GA, strategies with higher benefits are more likely to survive and generate new variants themselves. Strategies that do not derive profits tend to disappear over the generations. Communication between the different species (or agents) is assumed to be confined solely to the development of the auction. In the proposed model (see the general scheme in Fig. 1), the fitness value corresponds to the profit obtained by the assessed strategy against the candidate strategies of the rest of the participants.

Once the model has been designed and implemented, private values of each species or bidders are established according a uniform distribution from the scenarios described in Section 3. The populations of strategies are initialized randomly, and the different species are allowed to co-evolve over a sufficient amount of time to enable them to analyze the behavior of different agents and auction mechanisms.

For purposes of replication, below is a list of the steps describing in more detail how the proposed co-evolutionary system works:

(1) Create as many species as number of bidders $(M)$. Each species $j$ has an independent population of individuals (strategies), and $P_{j, n}$ represents the individual $n$ from the species $j$, where $1 \leq j \leq M$ (number of species/bidders) and $1 \leq n \leq P_{\text {size }}$ (population size).

(2) Randomly initialize from a uniform distribution all populations of strategies for all species. In the proposed system, each individual has one chromosome $C$ with 10 genes $(g)$, and each gene can encode integer values from 1 to 10. Thus, one individual/chromosome represents 10 different possible strategies: $P_{j, n}=C_{j, n}^{1}=\left[g_{j, n}^{1}, g_{j, n}^{2}, . ., g_{j, n}^{10}\right]$, where $g_{j, n}^{x} \in\{1,2,3,4,5,6,7,8,9,10\}$. This process of chromosome encoding is similar to the one used in Mochon et al. (2009, 
2011). Basically, these values tell the buyer how to bid, meaning the percentage of his/her value that he/she should report. In this work, the bid is calculated using Eq. (5):

$$
b_{j, n}^{x}=\frac{\left(g_{j, n}^{x}\right)}{10} \times v_{j}(S)
$$

(3) Evaluate all the individuals from all the species. For this step, starting from the first bidder, each individual/strategy of the population is evaluated against the best-valued strategy from the others (called candidate strategy or representative individual from the species). During the first iteration, as there will be no prior information about which are the best strategies, the first individual of each population will be selected as representative $\left(P_{j, n}^{\text {best }}=P_{j, 1}\right)$.

(4) A complete cycle of evaluations is concluded when all the strategies from all the species are evaluated and evolve (generation):

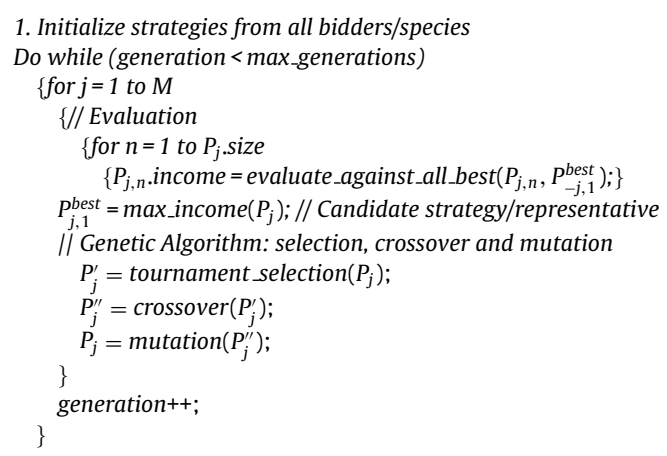

(5) After evaluating all the strategies from all the species against the best ones from the others (bidders/species, $P_{-j, 1}^{\text {best }}$ ), each independent GA tries to optimize its population of strategies using the profit of the bidder $j$ obtained when applying strategy $n$ as the fitness value. The best strategies will have a greater chance to survive and reproduce. First, the tournament selection will run several "tournaments" among a few individuals chosen at random from the entire population. The size of the tournament can be changed and determines the selection pressure. Each "tournament" will have a winner (the one with the best fitness value) that will be selected as parent for the next steps. Then, uniform crossover and mutation will create the new generation. The crossover operator is in charge of mixing the parents' genetic code. The uniform crossover chooses randomly with a probability of $50 \%$ between the genetic code of both parents. Therefore, the offspring will have approximately half of the genes from the first parent and the other half from the second one. Once the uniform crossover has finished, then a percentage (i.e. 10\%) of the new genetic material is randomly changed. This genetic operator plays a key role in evolution since it preserves genetic diversity between individuals and from one generation to the next. In addition, it allows the algorithm to avoid local solutions and to find and improve new solutions from the old ones. The best individual, referred here as the candidate strategy or representative individual, will be part of the new generation. This is commonly known as elitism.

(6) This cycle will iterate as many times as the maximum number of generations (max generations). Traditionally when working with GAs, the stop criteria relate to number of generations and convergence (i.e., which occurs if the fitness value is improving). However, in the case of co-evolution, stopping is much more complicated. In this type of game, a strategy that was good in the past may not be good in the future, depending on the strategies and preference structure of the other bidders, as all bidders are dynamically searching for profit maximizing strategies. When dealing with this type of problem, it is desirable to run the model for many generations to obtain a sample large enough to observe improved strategies, equilibriums or bidding patterns. In addition, as GAs are stochastic algorithms, experiments must be successfully repeated several times before drawing conclusions.

(7) Finally, the co-evolutionary system developed, after a round of complete assessments (in which all species have been evaluated) is finalized, which updates the set of bidder preferences. This change follows a uniform distribution that is sampled as many times as the number of generations (max generations) between two limits that represent the range of possible values that a bidder can have. These values are randomly chosen during the first experiment and are then used for the remainder of the experiments to guarantee the same conditions for a sequence of experiments. There are two main reasons for this continuous changing: a) to avoid bidders' learning their competitors' preferences and b) to incorporate uncertainty into the model. This uncertainty pushes bidders to seek improved strategies that are tested and analyzed under different scenarios.

\subsection{Experimental setup}

Each experiment consists of ten executions that simulate the behavior of the participants by means of the co-evolutionary system. Nine different experiments are needed: three scenarios (symmetric, asymmetric and strong asymmetric) tested with three pricing mechanisms (first-price, VCG and BPO). During the simulations, the partial results obtained from the encounters 
Table 5

Adjustment parameters of the genetic algorithm.

\begin{tabular}{ll}
\hline Parameters/operators & Values \\
\hline Number of generations & 1000 \\
Population size & 50 \\
Length of the chromosome & 10 \\
Alleles & 1.10 \\
Selection operator & Tournament of $10 \%$ \\
Crossover operator & Uniformity of $100 \%$ \\
Mutation factor & $10 \%$ \\
Elitism & 1 individual \\
Replacement policy & Generational of $100 \%$ \\
\hline
\end{tabular}

among the best individuals of each species (representative individuals) are stored. As described above, to determine which individuals should represent each species, the co-evolutionary system implements an independent GA for each species, and to assess a population, the best individuals of the other species are used. The proposed system co-evolves for 1000 iterations for each simulation, which yields a sufficiently large sample size to obtain statistically significant results. As described before, a generation requires a full iteration of all populations of the species, which means between 150,000 and 200,000 evaluations (depending on the scenario), and for each experiment $(10 \times 1000 \times$ Psize $\times$ bidders). All GA parameters listed in Table 5 have been determined empirically and are based on experiences gathered from previous works (Mochon et al., 2007, 2011), where each independent population consists of 50 individuals represented by vectors of 10 strategies, with integer values in the range [1...10]. Nevertheless, different parameters were tested for the population size (25 and 100), the rates of mutation (1-5\%), and the selection operator with tournaments of $5 \%$ (less selective pressure) and roulette wheel. In all cases tested, only minor differences were found in the results, and the effects produced by the parameters in the simulation were similar and proportional for all scenarios and pricing rules. It must be taken into account that all parameters are the same for all species, and as a result, the evolution of all will be affected in the same manner (faster or slower).

Finally, it is important to highlight that the aim of this co-evolutionary system is not to obtain equilibrium strategies but rather to find dynamic improved strategies that survive after many executions (million of auctions each experiment) whereby all bidders try to maximize their profits. Our aim is to generate a benchmark for analysis that helps to understand how to deal with the complications of real markets where theoretical results are not available. ${ }^{14}$

\section{Computational experiments}

This section discusses the strategies followed by the participants with the three pricing rules implemented and the final outcome in terms of allocation and payments.

\subsection{Bidders' strategic behavior}

Reeves et al. (2005) pointed out that finding equilibrium strategies for complex games such as ours is extremely complicated. Discovering optimal strategies by enumeration is infeasible and best-response behavior is highly sensitive to preference structure and rivals' strategies. Hence, we propose a co-evolutionary system as a method for finding improved strategies, i.e., strategies that survive along evolution and thus prove their robustness, even when uncertainty is present. As Miller (1996) denoted, the use of artificial adaptive techniques can create a benchmark for analysis that complements results from traditional theory.

To analyze bidders' behavior, Fig. 2 exhibits the improved strategies histogram that the co-evolutionary system proposes (the $x$-axis represents the $g_{j, 1}^{\text {best }} / 10$ value). This figure shows the strategies collected from nine arbitrarily-selected simulations of 1000 auctions played by the best evolved individuals (representative bidders) of each cycle $\left(P_{s, n}^{b e s t}\right)$, displayed by bidder, scenario and pricing rule.

With the first-price rule, bidders tend to shade their bids in all the scenarios, although this strategy is affected by the existence of asymmetries as well as the bidders' type. Bid shading is more significant when bidders are symmetric. In this scenario, bidders' leading strategies are to bid only for $20 \%$ or $30 \%$ of their real value. As competition increases and asymmetric bidders are included, they continue underbidding but not as strongly. In the second scenario, participants tend to bid between $40 \%$ and $50 \%$ of their real value. Furthermore, if the scenario involves strong asymmetries, although bidders continue underbidding, weak bidders bid more aggressively to try to earn some lots. Weak bidders report about $70 \%$ or $80 \%$ of their personal value while strong bidders report between $50 \%$ and $60 \%$.

\footnotetext{
${ }^{14}$ Regarding to the power of the used procedure, we have previously tested it within different known simple scenarios. The observations made under these simple test cases were convincing and corroborate other works. All the bidding behaviour gathered from the virtual bidders was plausible. Furthermore, our results also corroborate the only theoretical framework within our mode, i.e., when the VCG mechanism is implemented the co-evolutionary system suggest bidding sincerely.
} 


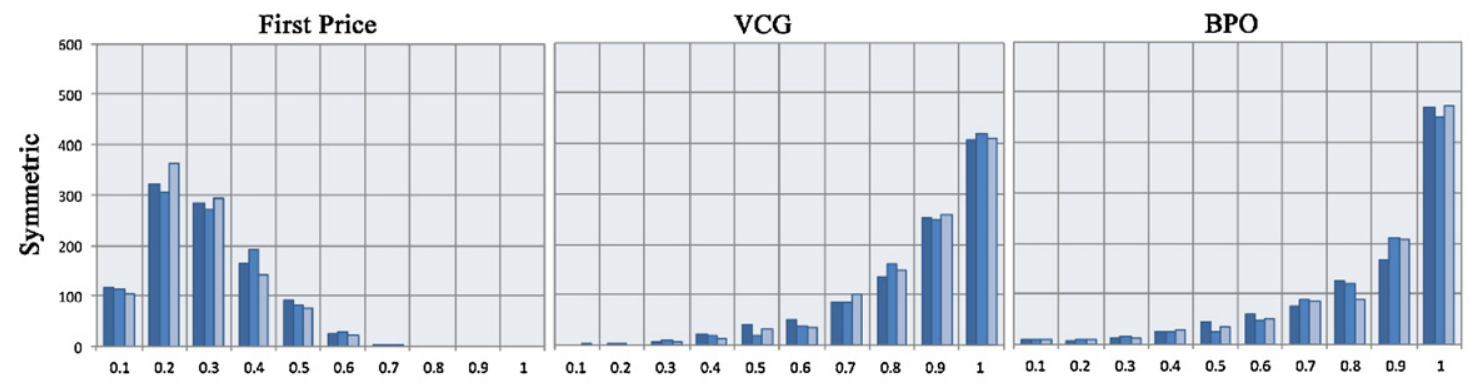

Bidder 1

- Bidder 2

$\square$ Bidder 3

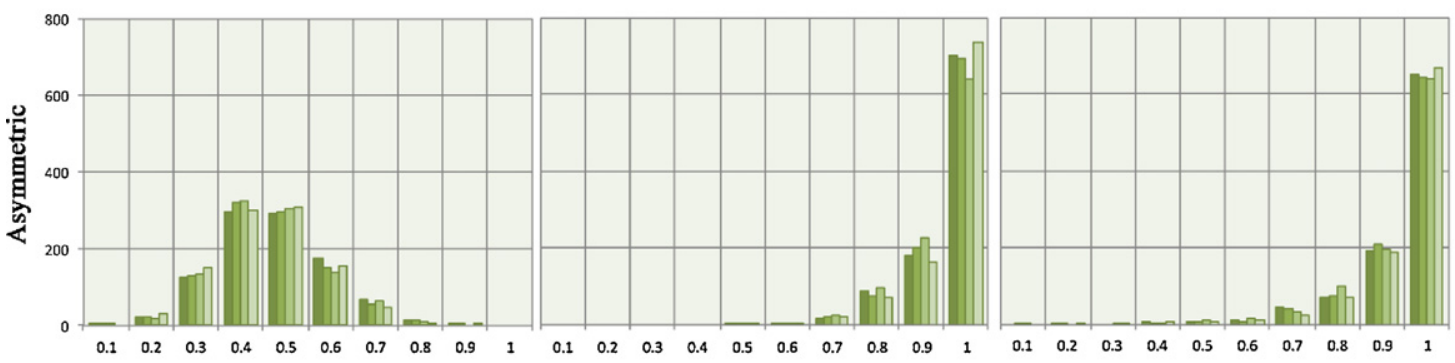

Bidder 1

- Bidder 2

$\square$ Bidder 3

$\square$ Bidder 4

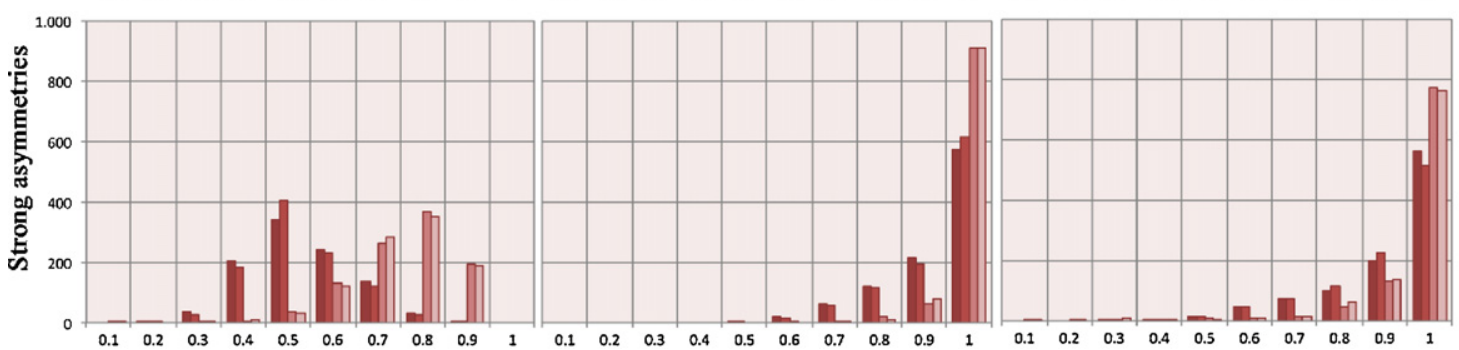

- Bidder 1

- Bidder 2

$\square$ Bidder 3

$\square$ Bidder 4

Fig. 2. Bidders' improved strategies histogram proposed by the co-evolutionary system per bidder, scenario and pricing rule.

When the VCG mechanism is implemented, the leading strategy used by participants is sincere bidding $\left(g_{j, 1}^{\text {best }} / 10=1\right)$ in all scenarios tested, and particularly in the presence of asymmetries. When participants are symmetric, they perform truthful bidding about $40 \%$ of the time. The frequency of this strategy when bidders are asymmetric is about $70 \%$. It is worth mentioning that when bidders exhibit strong asymmetries, weak bidders learn very quickly that their best strategy is truthful bidding, and then they follow it $90 \%$ of the time, whereas strong bidders do so around $60 \%$ of the time. These results corroborate theoretical findings that demonstrate that the VCG mechanism is incentive compatible and sincere bidding is the dominant strategy for all participants. In our system, this behavior turns out to be all bidders' improved strategy. ${ }^{15}$

The behavior proposed by the co-evolutionary system in the core-selecting auction is similar to that observed for the VCG mechanism, but with some differences worthy of comment. When bidders are symmetric and the BPO mechanism is implemented, bidders bid sincerely with a frequency slightly higher than that with the VCG mechanism (around $47 \%$ of the time, compared with $40 \%$ for the VCG mechanism). Nevertheless, the opposite happens in the presence of asymmetries. In the second scenario, bidders follow this strategy about $65 \%$ of the time. Finally, if there are strong asymmetries among bidders, the frequency with which weak participants bid sincerely is around $75 \%$, and for strong ones it is around $55 \%$.

\subsection{Results in terms of the allocation of lots}

When participants bid according to their true values, an efficient allocation of lots is produced, regardless of the price mechanism selected. However, when there is no sincere bidding, the selection of a particular pricing rule will affect the bidding behavior of participants and thus the final allocation of the lots. Table 6 gathers the spectrum amount (in $\mathrm{MHz}$ ) that each bidder wins under the different pricing rules when they bid strategically according to the developed co-evolutionary system as well as the efficient allocation for each simulated scenario. This table also includes the differences (in percentages) with respect to the efficient allocation.

As can be seen from the table, major differences with respect to the efficient allocation appear when implementing the first-price rule, and these differences are statistically significant ${ }^{16}$ for all bidders and scenarios. When there are strong

\footnotetext{
${ }^{15}$ Because GAs are stochastic techniques with random mutation, the strategies are forced to change during the process.

${ }^{16}$ For $p<0.05$
} 
Table 6

Spectrum allocated with the co-evolutionary system to bidder 1 (B1), bidder 2 (B2), bidder 3 (B3) and bidder 4 (B4) in each scenario with the different pricing rules tested.

\begin{tabular}{|c|c|c|c|c|c|c|c|c|c|}
\hline & $\mathrm{B} 1(\mathrm{MHz})$ & Dif $^{*}$ & $\mathrm{~B} 2(\mathrm{MHz})$ & Dif $^{*}$ & B3 (MHz) & Dif $^{*}$ & $\mathrm{~B} 4(\mathrm{MHz})$ & Dif $^{*}$ & Total $(\mathrm{MHz})$ \\
\hline \multicolumn{10}{|c|}{ Symmetric scenario } \\
\hline COEV_1st & 26.52 & $3.43 \%{ }^{* *}$ & 26.89 & $3.57 \%$ & 26.49 & $0.82 \%$ & - & - & 79.91 \\
\hline COEV_VCG & 25.16 & $-1.87 \%$ & 25.92 & $-0.19 \%$ & 26.87 & $2.25 \%^{* *}$ & - & - & 77.95 \\
\hline COEV_BPO & 25.36 & $-1.06 \%$ & 26.24 & $0.98 \%$ & 26.50 & $0.85 \%$ & - & - & 78.09 \\
\hline Efficient & 25.64 & - & 25.97 & - & 26.28 & - & - & - & 77.89 \\
\hline \multicolumn{10}{|c|}{ Asymmetric scenario } \\
\hline COEV_1st & 21.06 & $4.44 \%^{* *}$ & 20.68 & $4.28 \%^{* *}$ & 19.24 & $-2.28 \%$ & 19.02 & $-5.49 \%^{* *}$ & 80.00 \\
\hline COEV_VCG & 20.22 & $0.24 \%$ & 20.14 & $1.53 \%$ & 19.70 & $0.04 \%$ & 19.86 & $-1.31 \%$ & 79.90 \\
\hline COEV_BPO & 20.78 & $3.01 \%{ }^{* *}$ & 19.86 & $012 \%$ & 19.61 & $-0.41 \%$ & 19.57 & $-2.74 \% *$ & 79.81 \\
\hline Efficient & 20.16 & - & 19.83 & - & 19.68 & - & 19.31 & - & 79.80 \\
\hline \multicolumn{10}{|c|}{ Strong asymmetries scenario } \\
\hline COEV_1st & 18.90 & $-33.78 \% * *$ & 19.00 & $-33.31 \%^{* *}$ & 21.13 & $84.04 \%^{* *}$ & 20.98 & $85.43 \%{ }^{* *}$ & 80.00 \\
\hline COEV_VCG & 26.68 & $-6.50 \%^{* *}$ & 26.78 & $-5.98 \%^{* *}$ & 13.38 & $16.52 \%{ }^{* *}$ & 13.14 & $16.12 \%{ }^{* *}$ & 79.98 \\
\hline COEV_BPO & 26.48 & $-7.20 \%{ }^{* *}$ & 26.29 & $-7.72 \%^{* *}$ & 13.69 & $19.23 \%{ }^{* *}$ & 13.44 & $18.81 \% * *$ & 79.90 \\
\hline Efficient & 28.53 & - & 28.48 & - & 11.48 & - & 11.31 & - & 79.81 \\
\hline
\end{tabular}

* Difference with respect to the efficient allocation.

** Statistically significant differences $(p<0.05)$.

Table 7

Average revenue of the seller in $\mathrm{M}$ Euros (SD in parentheses).

\begin{tabular}{|c|c|c|c|c|c|c|}
\hline & $R_{\text {COEV }}^{1 s t}$ & $R_{C O E V}^{V C G}$ & $R_{C O E V}^{B P O}$ & $R_{S B}^{V C G}$ & $\begin{array}{l}\text { Full-information } \\
\text { Benchmark Revenue }\left(R_{S B}^{B P O}\right)\end{array}$ & Optimal value $\left(R_{S B}^{1 \mathrm{st}}\right)$ \\
\hline Symmetric scenario & $60.16(15.85)$ & $73.18(17.52)$ & $75.72(17.34)$ & $83.92(17.61)$ & $87.43(15.78)$ & $188.49(9.46)$ \\
\hline Asymmetric scenario & $100.31(15.89)$ & $146.23(18.98)$ & $143.15(21.99)$ & $155.26(17.26)$ & $156.85(16.03)$ & $200.71(8.57)$ \\
\hline Strong asymmetries scenario & $110.84(11.19)$ & $122.66(8.22)$ & $120.91(10.68)$ & $125.18(7.14)$ & $127.44(8.20)$ & $173.73(5.93)$ \\
\hline
\end{tabular}

The superscripts are used to differentiate between the different pricing rules (1st, VCG and BPO) and the subscripts to differentiate among the bidding behavior (sincere bidding, SB, and co-evolutionary system, COEV).

asymmetries among participants, the differences are statistically significant regardless of the pricing rule selected. Still, these differences are much more pronounced under the first-price mechanism, whereas the VCG and BPO mechanisms yield a final allocation closer to that of the efficient one.

These results are a consequence of the strategies discussed in the previous subsection. With the first-price rule, participants bid below their values to obtain positive profits. Determining how much to bid below their values depends primarily on their predictions of the bids of their rivals. ${ }^{17}$ Each participant will try to make the lowest possible bid that will still be the highest among all those entered. This decision involves making bids with significant deviations from true values. Therefore, the final award ceases to be efficient as the participant with the highest value is not necessarily the one who makes the highest bid.

When the VCG mechanism is implemented, the co-evolutionary system proposes sincere bidding as the leading strategy, giving a final allocation close to the efficient one. With this mechanism, differences with respect to the efficient allocation are only significant in the strong asymmetries scenario. ${ }^{18}$ The core-selecting auction (BPO mechanism) realizes nearly the same efficiency as does the VCG mechanism, and the differences between these two mechanisms are not statistically significant for $p<0.05$.

\subsection{Results in terms of final payments}

The average revenues of the seller generated by each mechanism for the three scenarios studied have also been analyzed, see Table 7. Following the terminology used by Hoffman et al. (2006), the optimal value of the auction is equal to the revenue of the seller if all participants bid according to their true values and if the first-price rule is established $\left(R_{S B}^{1 s t}\right)$. However, with this pricing-rule participants never bid sincerely. Therefore, the full-information benchmark revenue that is generated, as a reference point, is equal to the payments that would be generated if all participants bid according to their true values under the BPO mechanism $\left(R_{S B}^{B P O}\right)$.

If all participants bid according to their true values, the results would remain in the expected order in which the BPO mechanism revenues fall at an intermediate point between those generated by the VCG and by the first-price mechanisms:

$$
R_{S B}^{V C G} \leq R_{S B}^{B P O} \leq R_{S B}^{1 \mathrm{st}}
$$

\footnotetext{
${ }_{17}$ Risk aversion may also affect this evaluation of how much to shade, but here we assume risk neutral bidders in all cases.

18 For $p<0.05$.
} 


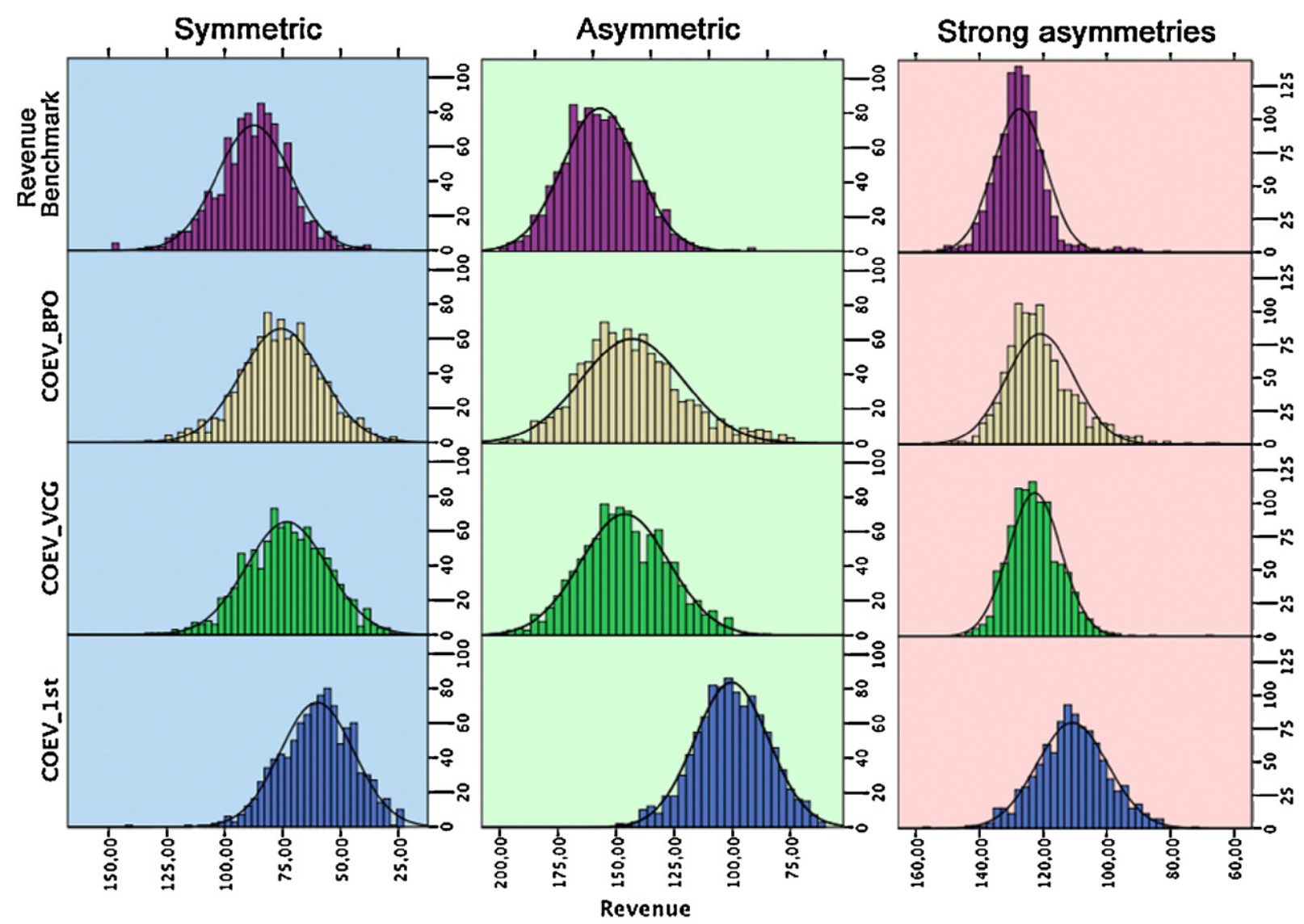

Fig. 3. Revenues histogram per scenario and pricing rule. Revenues obtained with the co-evolutionary system (COEV) with the different pricing rules (1st, VCG and BPO) for each scenario (symmetric, asymmetric and strong asymmetries) compared to the full-information benchmark revenue, $R_{S B}^{B P O}$ (in $M$ Euros). $X$-axis is inverted.

For a more detailed analysis of the revenues with respect to the full-information benchmark revenue, Fig. 3 depicts the revenues histogram obtained with the co-evolutionary system for each scenario and pricing rule. In examining revenues, if the participants maintain the strategic behavior of the co-evolutionary system, we see that the first-price mechanism achieves the lowest revenue as a consequence of the significant bid shading described previously. This situation is true for all scenarios but differences are most pronounced in the asymmetric scenario where average and strong bidders are included. ${ }^{19}$

In contrast, with the VCG and BPO mechanisms the co-evolutionary system motivates participants to report true values as their leading strategy, and so the final revenues are closer to the full-information benchmark revenue, although we still find some differences. It is worth noting that differences are more significant in the symmetric scenario, whereas revenues obtained with both mechanisms in the presence of strong asymmetries are closer to the full-information benchmark revenue. These results are consistent with the analysis of the strategic behavior done at the beginning of this section, as the truthful bidding is more intense when asymmetries exist. Fig. 4 shows the revenue percentage that the seller loses compared with the full-information benchmark revenue $\left(R_{S B}^{B P O}\right)$ with each pricing rule for every scenario when bidders follow the co-evolutionary system. As mentioned, the most significant differences relative to the benchmark case are found when the participants follow the first-price rule. Therefore, if the seller seeks to maximize the payments that are to be made by the participants, he should opt for a VCG or BPO mechanism before a first-price mechanism. ${ }^{20}$

Given a set of private values for each bidder, the final outcome that will be obtained with the VCG and BPO mechanisms depends on the strategies played (reported values) and on the blocking coalitions that appear. If no coalitions block the result obtained by the VCG, the two mechanisms generate the same revenue for the seller with respect to the reported

\footnotetext{
19 In all scenarios, the differences found between the revenues obtained with the first-price rule when the participants follow the co-evolutionary system and the full-information benchmark revenue are statistically significant for $p<0.05$.

20 The differences between revenues generated when the participants follow the co-evolutionary system with the first-price rule are also statistically significant respect to revenues obtained when participants follow the co-evolutionary system but under the VCG or BPO mechanism for all scenarios, considering $p<0.05$.
} 


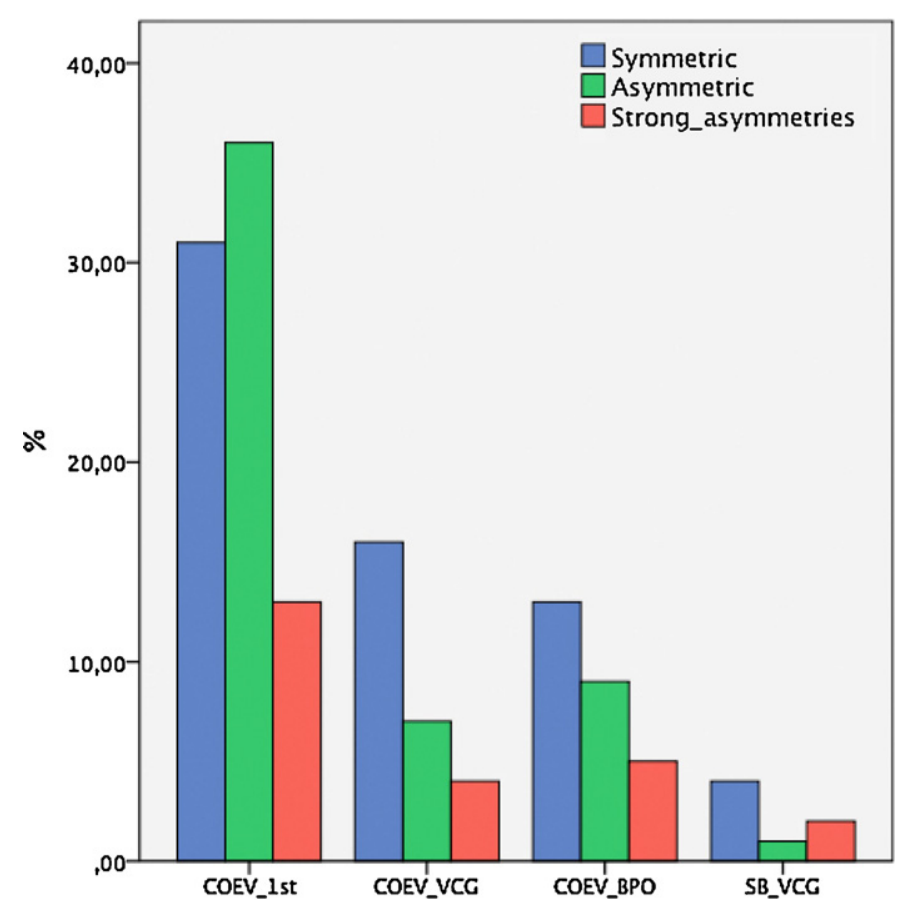

Fig. 4. Percentage of the average revenues that the seller loses with the co-evolutionary system (COEV) with each pricing rules (1 st, VCG and BPO) in each scenario (symmetric, asymmetric and strong asymmetries) compared to the full-information benchmark revenue, $R_{S B}^{B P O}$.

Table 8

Analysis of blocking coalitions in 1000 experiments per scenario.

\begin{tabular}{|c|c|c|c|c|}
\hline & \multicolumn{2}{|l|}{ Sincere bidding strategy } & \multicolumn{2}{|l|}{ Co-evolutionary strategy } \\
\hline & $\begin{array}{l}\% \text { of experiments with at } \\
\text { least one blocking coalition }\end{array}$ & $\begin{array}{l}\text { Average number of } \\
\text { iterations }\end{array}$ & $\begin{array}{l}\% \text { of experiments with at } \\
\text { least one blocking coalition }\end{array}$ & $\begin{array}{l}\text { Average number of } \\
\text { iterations }\end{array}$ \\
\hline Symmetric scenario & $40.9 \%$ & 2.27 & $41.5 \%$ & 2.23 \\
\hline Asymmetric scenario & $20.2 \%$ & 2.92 & $17.6 \%$ & 2.50 \\
\hline Strong asymmetries scenario & $43.9 \%$ & 2.79 & $44.8 \%$ & 2.72 \\
\hline
\end{tabular}

values. However, if the VCG mechanism does not provide a core outcome, the BPO imposes core constraints to achieve some fair payments, increasing the seller's revenues. Table 8 displays the number of experiments in which there was at least one blocking coalition as well as the average number of iterations performed to reach a core outcome in the coreselecting auction. In all simulated scenarios, a significant percentage (from $17.6 \%$ to $44.8 \%$ ) of occasions exists where the VCG mechanism does not generate a core outcome in the sincere bidding and the co-evolutionary case. ${ }^{21}$

Nevertheless, we cannot conclude that the BPO always yields higher revenues than the VCG does, as we found differences in the strategy played by the participants in each mechanism. As mentioned, the frequency of sincere bidding obtained with the co-evolutionary system is slightly higher in the BPO mechanism than in the VCG in the symmetric scenario, whereas the opposite happens in the asymmetric scenarios. Hence, in the experiments conducted, when participants behave according to the co-evolutionary system, the core-selecting auction obtains, on average, $3.46 \%$ higher revenues than does the VCG mechanism when bidders as symmetric. Nevertheless, the VCG mechanism obtains $2.15 \%$ higher average revenues than does the BPO mechanism in the asymmetric scenario and $1.45 \%$ higher average revenues in the strong asymmetric scenario despite the existence of coalitions.

To analyze these results in more depth, we focused on those experiments in which a significant number of coalitions block the outcome generated by the VCG mechanism. We included those experiments in which the BPO identifies simultaneously three or more blocking coalitions in the sincere bidding and the co-evolutionary case. ${ }^{22}$ Table 9 shows the average revenues

\footnotetext{
${ }^{21}$ It is important to note that the existence of a greater or lesser number of blocking coalitions depends on the structure of the bids made by participants. In other simulation environments tested (although not included in this study), situations have been found in which this situation was barely present, so that differences between the two mechanisms did not reach statistical significance.

${ }^{22}$ We also analyzed those experiments for which the sincere bidding case of BPO (and not the co-evolutionary BPO) identifies three or more blocking coalitions and those for which the co-evolutionary case of the BPO (and not the sincere bidding BPO) identifies three or more blocking coalitions. In all this testing, no significant differences were found in the results.
} 
Table 9

Average revenue of the seller in M Euros for those experiments in which the BPO mechanism detects simultaneously three or more blocking coalitions in the sincere bidding and the co-evolutionary case (SD in parentheses).

\begin{tabular}{lrrr}
\hline & $R_{\text {COEV }}^{V C G}$ & $R_{\text {COEV }}^{B P O}$ & $\begin{array}{l}\text { Full-information } \\
\text { Benchmark Revenue }\left(R_{S B}^{B P O}\right)\end{array}$ \\
\hline Symmetric scenario & $58.01(11.30)$ & $75.55(17.02)$ & $86.03(20.92)$ \\
Asymmetric scenario & $129.46(17.96)$ & $143.77(20.85)$ & $152.89(18.54)$ \\
Strong asymmetries scenario & $119.75(7.78)$ & $124.15(9.15)$ & $128.04(8.53)$ \\
\hline
\end{tabular}

The superscripts are used to differentiate between the different pricing rules (1st, VCG and BPO) and the subscripts to differentiate among the bidding behavior (sincere bidding, SB, and co-evolutionary system, COEV).

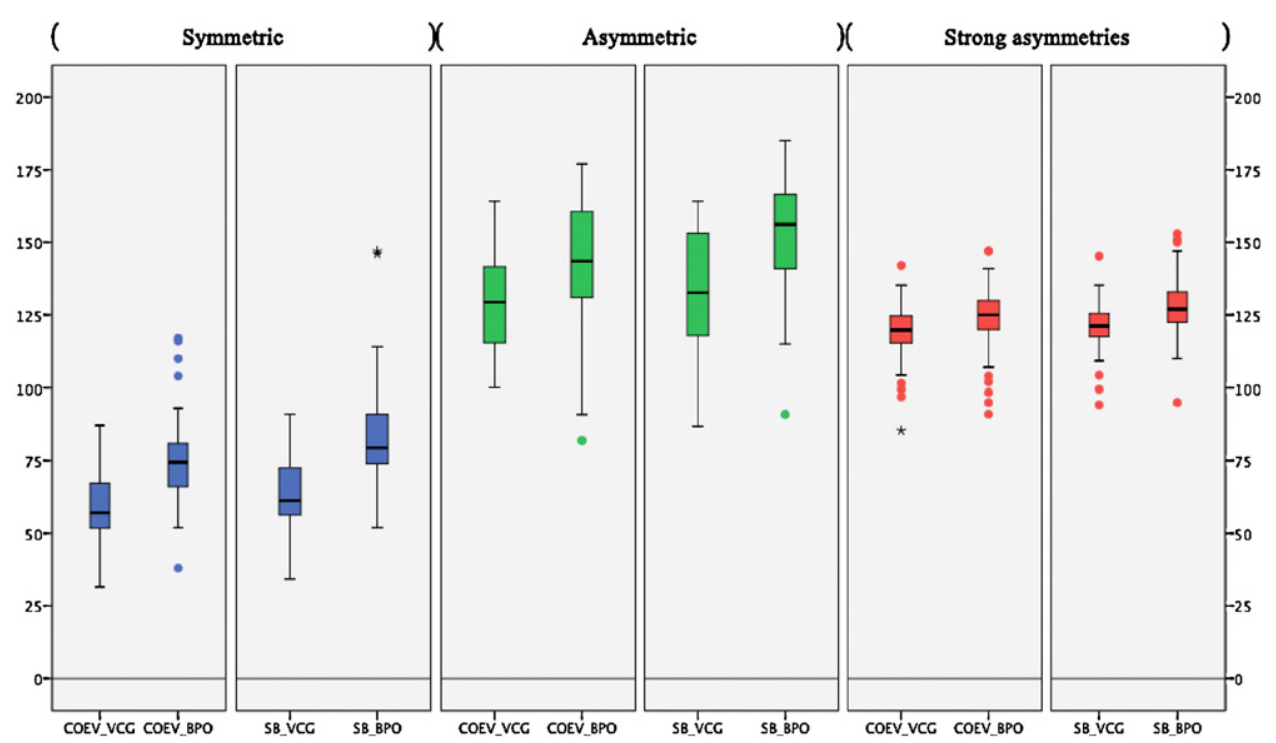

Fig. 5. Box plot with the revenue when the VCG and the BPO pricing rules are implemented for those cases in which the BPO mechanism detects simultaneously more than three blocking coalitions both in the sincere bidding case and the co-evolutionary case (M Euros).

obtained with these two pricing mechanisms, the full-information benchmark revenue and the optimal value. In these experiments, regardless of the bidding strategy, the core-selecting auction always yields greater revenues than does the VCG mechanism, thus satisfying the following order:

$$
R_{C O E V}^{V C G} \leq R_{C O E V}^{B P O} \leq R_{S B}^{B P O}(\text { full - information benchmark revenue }) \leq R_{S B}^{1 \text { st }}(\text { optimal value })
$$

Likewise, the differences found between the payments to be made by participants with the two pricing rules are statistically significant in all scenarios. ${ }^{23}$ For further analysis the box plot in Fig. 5 depicts the differences among revenues generated with both pricing rules for the scenarios considered when three or more blocking coalitions are present simultaneously with the sincere bidding strategy and the co-evolutionary behavior. The risk that the VCG mechanism may generate results that are not in the core is an important drawback of this mechanism.

It is important to note that, regardless of the mechanism selected, if participants bid according to the strategies proposed by the co-evolutionary system, significant differences exist between generated revenue and the full-information benchmark revenue for all pricing rules and environment tested. ${ }^{24}$ One possible solution to prevent this loss of revenue is to use a combinatorial auction with multiple rounds such as the clock-proxy auction proposed by Ausubel et al. (2006).

\section{Concluding remarks}

In recent years, combinatorial auctions have become an essential tool in awarding multiple related items in both businessto-business and governmental auctions in complex markets. Nevertheless, theoretical analysis of combinatorial auctions in the presence of complementarities, private information and asymmetries can be extremely difficult. The main contribution of this paper is to recourse to computational economics to advance the analysis of intricate environments for which theoretical results are not available.

\footnotetext{
23 For $p<0.01$.

24 For $p<0.05$
} 
In this paper, we have analyzed the effects of the choice of a particular pricing mechanism on the final outcome in a combinatorial sealed-bid auction. The studied pricing rules are the first-price mechanism, the VCG mechanism and the BPO mechanism (a specific core-selecting auction). For the purpose of studying these pricing rules, we simulated three real-life case scenarios for the sale of spectrum licenses. These scenarios include situations with both symmetric and asymmetric participants, with private information and a complex preference structure involving substitutes and complements items. To simulate the behavior of the participants, we developed an agent-based co-evolutionary model that seeks to maximize the profit of each agent. This system yields improved strategies that survive along evolution, providing a benchmark for analyzing each scenario.

In light of the results obtained, out first conclusion is that, in a combinatorial sealed-bid auction with the pay-your-bid rule, bidders shade their bids in an effort to maximize expected profits. This behavior implies a significant revenue reduction as well as an inefficient allocation of items that is heightened when the scenario includes asymmetries.

In contrast, in both the VCG and the BPO mechanisms, the co-evolutionary system motivates sincere bidding as the leading improved strategy, and this strategy is particularly favored when the scenario involves asymmetries and competition increases. Nevertheless, the sincere bidding strategy is more intense in the BPO than in the VCG mechanism when bidders are symmetric, and the opposite is true for the asymmetric scenarios. Hence, we observe that, if participants follow the strategic behavior determined by the co-evolutionary system, the BPO mechanism yields greater revenue on average when bidders are symmetric, but when bidders are asymmetric, the average revenue obtained with the VCG mechanism is higher.

The main problem that we encounter by selecting the VCG mechanism is that it often yields payments not in the core, thus generating socially unacceptable situations. By limiting the study only to cases where the BPO mechanism finds simultaneously a significant number of blocking coalitions with the sincere bidding and the co-evolutionary behavior, the core-selecting auction always obtains higher revenues than does the VCG mechanism, and these differences are statistically significant in all scenarios. Another reason for policymakers to implement a core-selecting auction is to avoid the major disadvantages of the VCG mechanism cited by Ausubel and Milgrom (2006), including low revenues, non-monotonic outcome and collusive bidding.

\section{Acknowledgements}

The authors want to thank the editor and referees for their helpful comments. This article has been financed by the Spanish research MCyT projects MSTAR, Ref: TIN2008-06491-C04-03/TIN and MOVES, Ref: TIN2011-28336.

\section{References}

Andreoni, J., Miller, J.H., 1995. Auctions with artificial adaptive agents. Games and Economic Behaviour 10, 39-64.

Ausubel, L.M., Cramton, P., McAfee, P., McMillan, J., 1997. Synergies in wireless telephony: evidence from the broadband PCS auctions. Journal of Economics and Management Strategy 6 (3), 497-527.

Ausubel, L.M., Cramton, P., Milgrom, P., 2006. The clock-proxy auction: a practical combinatorial auction design. In: Cramton, P., Shoham, Y., Steinber, R. (Eds.), Combinatorial Auctions. MIT Press, USA, Cambridge, pp. 115-138.

Ausubel, L.M., Milgrom, P., 2002. Ascending auction with package bidding. Frontiers Theoretical Economics 1 (1), 1-42.

Ausubel, L.M., Milgrom, P., 2006. The lovely but lonely Vickrey auction. In: Cramton, P., Shoham, Y., Steinber, R. (Eds.), Combinatorial Auctions. MIT Press, Cambridge, USA, pp. 17-40.

Ausubel, L.M., Baranov, O.V., 2010. Core-selecting auctions with incomplete information, Working Paper, University of Maryland.

Avenali, A., Bassanini, A., 2007. Simulating combinatorial auctions with dominance requirement and loll bids through automated agents. Decision Support Systems 43 (1), 211-228.

Avenali, A., 2009. Exploring the VCG mechanism in combinatorial auctions: the threshold revenue and the threshold-price rule. European Journal of Operational Research 199, 262-275.

Banks, J., Olson, M., Porter, D., Rassenti, S., Smith, V., 2003. Theory, experiment and the federal communications commission spectrum auctions. Journal of Economic Behavior \& Organization 51 (3), 303-350.

Becker, L., Seshadri, M., 2003. Cooperative coevolution of technical trading rules, Technical report, Worcester Polytechnic Institute.

Byde, A., 2002. Applying evolutionary game theory to auction mechanism design. Technical Report HPL-2002-321, HP Laboratories.

Chen, S.-H., Wang, X., Wong, K.P., Chung, C.-Y., 2006. A framework of oligopolistic market simulation with coevolutionary computation. Lecture Notes in Computer Science, vol. 4221. Springer, Berlin/Heidelberg, pp. 860-869.

Chen, S.-H., Zeng, R.-J., Yu, T., 2009. Co-evolving trading strategies to analyze bounded rationality in double auction markets. In: Riolo, R., Soule, T., Worzel, B. (Eds.), Genetic Programming: Theory and Practice VI, pp. 195-213.

Chen, S.-H., Zeng, R.-J., Yu, T., 2009b. Analysis of micro-behavior and bounded rationality in n auction markets using co-evolutionary GP. In: Proceedings of the first ACM/SIGEVO Summit on Genetic and Evolutionary Computation, Shanghai, China, pp. 807-810.

Cliff, D., 1998. Evolving parameter sets for adaptive trading agents in continuous double auction markets. In: Workshop on Artificial Societies and Computational Markets, Minneapolis, USA, pp. 38-47.

Cliff, D., 2003. Explorations in evolutionary design of online auction market mechanisms. Electronic Commerce Research and Applications 2, $162-175$.

Clarke, E., 1971. Multipart pricing of public goods. Public Choice 11 (1), 7-33.

Dawid, H., 1999. On the convergence of genetic learning in a double auction market. Journal of Economic Dynamics and Control 23, $1545-1567$.

Day, R.W., Cramton, P., 2008. The quadratic core-selecting payment rule for combinatorial auctions, Working Paper, University of Maryland.

Day, R.W., Milgrom, P., 2008. Core-selecting package auctions. International Journal of Game Theory 36, $393-407$.

Day, R.W., Raghavan, S., 2007. Fair payments for efficient allocations in public sector combinatorial auctions. Management Science 53 (9), $1389-1406$.

Erdil, A., Klemperer, P., 2010. A new payment rule for core-selecting package auctions. Journal of European Economic Association 8 (2-3), 537-547.

Giordana, A., Neri, F., 1996. Search-intensive concept induction. Evolutionary Computation 3 (4), $375-416$.

Goerre, J. K., Lien, Y., 2009. On the impossibility of core-selecting auctions. IEW - Working Papers from Institute for Empirical Research in Economics, University of Zurich. 
Gomez-Barroso, J.L., Mochon, A., Saez, Y., Feijoo, C., 2011. The importance of the size of the digital dividend when digital dividend spectrum is auctioned. International Journal of Mobile Communications 9 (1), 57-75.

Groves, T., 1973. Incentives in teams. Econometrica 41 (4), 617-631.

Hart, P.E., Nilsson, N.J., Raphael, B., 1968. A formal basis for the heuristic determination of minimum cost paths. IEEE Transactions on Systems Science and Cybernetics SSC 4 (2), 100-107.

Hillis, W.D., 1990. Co-evolving parasites improve simulated evolution as an optimization procedure. Physica D: Nonlinear Phenomena 42 (1-3), $228-234$

Hoffman, K., Menon, D., Heever, V.D., Wilson, T., 2006. Observations and near-direct implementation of the ascending proxy auction. In: Cramton, P., Shoham, Y., Steinber, R. (Eds.), Combinatorial Auctions. MIT Press, USA, Cambridge, pp. 415-450.

Husbands, P., Mill, F., 1991. Simulated co-evolution as the mechanism for emergent planning and scheduling. In: Belew, R.K., Booker, L.B. (Eds.), Proceedings of the Fourth International Conference on Genetic Algorithms. Morgan Kaufmann, San Mateo, pp. $264-270$.

Lund, H.H., 1995. Specialization under social conditions in shared environments. In: Proceedings of the Third European Conference on Artificial Life ECAL'95. Springer-Verlag, pp. 447-489.

Miller, J.H., 1996. The coevolution of automata in the repeated prisoner's dilemma. Journal of Economic Behavior and Organization 29 (1), 87-112.

Mishra, D., Parkes, D., 2007. Ascending price Vickrey auctions for general valuations. Journal of Economic Theory 132 (1), $335-366$

Mochon, A., Saez, Y., Quintana, D., Isasi, P., 2007. Bidding with memory in the presence of synergies: a genetic algorithm implementation. In: Proceedings of the IEEE Congress on Evolutionary Computation, pp. 228-235.

Mochon, A., Saez, Y., Isasi, P., Gomez-Barroso, J.L., 2009. Testing bidding strategies in the Clock-Proxy auction for selling radio spectrum. A Genetic Algorithm approach. In: Proceedings of IEEE Congress on Evolutionary Computation, pp. 2348-2353.

Mochon, A., Saez, Y., Isasi, P., Gomez-Barroso, J.L., 2011. The clock proxy auction for allocating radio spectrum licenses. Computational Economics 37, $411-431$.

Nie, L., Xu, X., Zhan, D., 2008. Coevolutionary computation based iterative multi-attribute auctions. In: Enterprise Interoperability III, Part V. Springer, London, pp. 461-469.

Ofcom, 2006. Digital Dividend Review. Document Consulting on the Proposed Approach to the Award of the Digital Dividend Spectrum (470-862 MHz). United Kingdom Office of Communications, http://www.ofcom.org.uk/consult/condocs/ddr/statement/.

Parkers, D., Ungar, L., 2002. An ascending-price generalized Vickrey auction. In: Proc. 2002. Stanford Institute for Theoretical Economics Workshop on the Economics of the Internet, Stanford, CA.

Phelps, S., Parsons, S., McBurney, P., Sklar, E. 2002. Co-evolution of auction mechanisms and trading strategies: towards a novel approach to microeconomic design. In: Workshop on Evolutionary Computation in Multi-Agent Systems, GECCO-02, pp. 65-72.

Plott, C.R., Salmon, T.C., 2004. The simultaneous, ascending auction: dynamics of price adjustment in experiments and in the UK3G spectrum auction. Journal of Economic Behavior \& Organization 53 (3), 353-383.

Porter, D., Rassenti, S., Shobe, W., Smith, V., Winn, A., 2009. The design, testing and implementation of Virginia's NOx allowance auction. Journal of Economic Behavior \& Organization 69 (2), 190-200.

Potter, M.A., De Jong, K.A., 1995. Evolving neural networks with collaborative species. In: Oren, T.I., Birta, L.G. (Eds.), Proceedings of the 1995 Summer Computer Simulation Conference. The Society for Computer Simulation. San Diego, California, pp. 340-345.

Potter, M.A., De Jong, K.A., 2000. Cooperative coevolution. An architecture for evolving coadapted subcomponents. Evolutionary Computation 8 (1), 1-29.

Reeves, D.R., Wellman, M.P., MacKie-Mason, J.K., Osepayshvili, A., 2005. Decision Support Systems 39, 67-85.

Rosin, C.D., Belew, R.K., 1995. Methods for competitive co-evolution. Finding opponents worth beating. In: Eshelman, L. (Ed.), Proceedings of the Sixth International Conference on Genetic Algorithms. Morgan Kaufmann, San Francisco, California, pp. 373-380.

Roth, A.E., 2002. The economist as engineer: game theory, experimentation, and computation as tools for design economics. Econometrica 70 (4), $1341-1378$.

Rust, J., Miller, J., Palmer, R., 1993. Behavior of trading automata in a computerized double auction market. In: The Double Auction Market. Addison-Wesley, pp. 155-198.

Saez, Y., Quintana, D., Isasi, P., Mochon, A., 2007. Effects of a rationing rule on the Ausubel auction: a genetic algorithm implementation. Computational Intelligence 23 (2), 221-235.

Saez, Y., Mochon, A., Gomez-Barroso, J.L., Isasi, P., 2008. Testing BOI and BOB algorithms for solving the winner determination problem in radio spectrum auctions. In: Proceedings of the 8th International Conference on Hybrid Intelligent Systems, pp. 732-737.

Sandholm, T., 2002. Algorithm for optimal winner determination in combinatorial auctions. Artificial Intelligence 135, 1-54.

Tivnan, B.F., 2005. Coevolutionary dynamics and agent-based models in organization science. In: Proceedings of the 37th Conference on Winter Simulation. Winter Simulation Conference, Orlando, Florida, December 04-07, pp. 1013-1021.

Vickrey, W., 1961. Counterspeculation, auctions and competitive saled tenders. Journal of Finance 16 (1), 3-8.

Wen, F.S., David, A.K., 2001. Strategic bidding for electricity supply in a day-ahead energy market. Electric Power Systems Research 59 (3), 197-206.

Yao, X., Darwen, P., 2000. Genetic algorithms and evolutionary games. In: Barnett, W.A., Marks, R., Chiarella, C., Schnabl, H., Keen, S. (Eds.), Commerce, Complexity and Evolution. Cambridge University Press, pp. 313-333. 